\title{
Short Review on Recent Trends in Dispersing Nanoclay Particles into Polymer Matrices Using Supercritical Carbon Dioxide
}

\author{
*Voore Gurunadha Rao, **Dr.S.Kalahasti, \\ Physics Research Scholar, Assistant Professor, \\ Department of Physics, \\ Dravidian University Kuppam \\ Kakatiya University, Warangal
}

\begin{abstract}
Polymer/clay nanocomposites are a new class of emerging materials in the last decades.Clays is used as filler due to their availability and good properties. In the low content of filler they improve barrier and mechanical properties. Nanocomposite developments have already been introduced for the majority of engineering plastics. Utilizing organomodified montmorillonite (MMT) (commonly called "Nanoclay") to reinforce polymer-based composites have raised much attention to academic and industrial sectors due to the addition of small amount of nanoclay could considerably enhance the mechanical properties of pristine polymers. The aim of this paper is to give short review on recent trends in dispersing nanoclay particles into polymer matrices using supercritical carbon dioxide.It also focuses on properties enhancement reported by different researchers and the $\mathrm{scCO} 2$ process was reviewed future challenges for this emerging technology in engineering applications.
\end{abstract}

Keywords: dispersed nanocomposites,nanoclay, supercritical $\mathrm{CO} 2$ processing

\section{INTRODUCTION}

The term "nano" refers to nano particle size from 1 to 100 nanometers. The term "nanotechnology" was first introduced by Norio Taniguchi in 1974. Nanotechnology may be used to improve the taste and texture of food and for the production of packaging that maintain fresh product. [1] The primary function of packaging is to maintain the quality and safety of products during transport and storage period, as well as to extend its viability by preventing unwanted effect agents such as microorganisms, chemical contaminants, oxygen, moisture and light. By studing of recently published literature, it was clear that nanomaterials such as nano polymers are trying to replace conventional materials in food packaging. Nanocomposites are currently being used in a number of fields, and new applications are continuously being developed including mechanical and biomaterial devices, as well as various in solar and fuel cells applications. [2] Nanosensors can be used to prove the presence of contaminants, microtoxins and microorganisms in food. [1] Generally, the synthesis of polymer nanocomposites are done using solution chemistry, and this may raise serious concerns regarding air and water pollutions. Immediately, the 'green' method using supercritical carbon dioxide $(\mathrm{scCO} 2)$ has grabbed the attention of researchers who are responsible to synthesize polymer composites by non-hazardous routes. Extrusion processes would benefit from the use of $\mathrm{scCO} 2$ since the rationale of the extrusion process is to formulate, provide texture and shape molten polymers by forcing them through a die. $\mathrm{ScCO} 2$ has been used in several studies as a medium of clay dispersion in polymer matrix by providing a solvent-free fabrication route for nanocomposites. [2] Well dispersion or intercalation/exfoliation of nanoscale fillers (such as nano-CaCO3 and nano-clay) in polymer matrix is currently not easy to be achieved. Supercritical carbon dioxide $(\mathrm{ScCO} 2)$ has been reported to have great potential for facilitating the dispersion or intercalation/exfoliation of the nanoscale fillers in polymer matrix. [3] An environmentally benign process, [4] Supercritical fluids have a unique and valuable potential for the enhanced processing of many materials[5].Furthermore, it has more favorable interactions with polymers compared to other inert gases and has the ability to be dissolved in large quantities. It acts as a plasticizer, which modifies viscosity and interfacial properties of the polymer drastically. [2] Dispersed polymer/clay nanocomposites are of great interest because they can significantly improve the properties of existing polymeric materials. However, achieving a high level of clay dispersion has been a key challenge in the production of polymer/clay nanocomposites. [6]

\section{POLYMER/CLAY NANOCOMPOSITES}

A material consists of two or additional components or phase known as composite material. In fact, a composite 
material is envisioned to supply properties in more than each the matrix and reinforcement phases that don't seem to be potential in conventional materials like polymers, metals, ceramics etc. Composites are being created using various matrices; like metals, ceramics, carbon, glasses and polymers, with polymers being the foremost common.

This can be because of the actual fact that polymers provide properties like low density, smart wettability of reinforcements and good toughness and straightforward to mould to a desired form, however have lower strength and modulus compared to ceramics and metals [9]. By the addition of reinforcing fillers into polymer matrices, the base polymer properties Strength are greatly improved amongst the foremost exciting reinforcements are the fillers that have the potential of up the electrical, thermal and mechanical properties which were similar to polymer matrices properties.

During the rapid development in the materials science and technology, a lot of research has extensively undertaken on superior performance polymer composites for targeted applications in various fields. With the advantage of nano engineering and science, A good number of efforts and makes an attempt are created to develop superior chemical compound composites. The new category of composites that contain comparatively very small amounts $(<10 \%)$ of nanometer-sized particles known as nanocomposites (NC)

Usually particles have nano-scale dimensions a minimum of one dimension that they're going to be mixed in organic polymer matrices. The polymer matrix is that the continual section so the reinforcement nanoparticles represent the phase. The nanoparticles behaviors and properties sometimes commanded by the composite properties. In current period, there was hefty concern within the utilization of nanocomposites to reinforce behavior properties like mechanical, barrier and electrical polymer properties. Polymeric NC get the large choice of property enhancements, e.g., enhanced gas barrier properties, improved ultraviolet light resistance, bigger dimensional stability, increased strength and stiffness and decreased electrical conductivity. [10-13]

\section{SUPERCRITICAL CARBON DIOXIDE (SCCO2) - POLYMER/CLAY NANOCOMPOSITES}

Supercritical carbon dioxide (scCO2) possesses properties of gas and liquid with high diffusion rates and solubilizing characteristics, correspondingly. Changing these density-driven properties can be consummate by adjusting temperature and pressure, making it a tunable fluid. In addition, $\mathrm{CO} 2$ is inexpensive, nontoxic, non-combustible and chemically stable. Carbon dioxide reaches its critical point at $31.06^{\circ} \mathrm{C}$ and $7.38 \mathrm{MPa}$, exhibiting a critical density of about $0.466 \mathrm{~g} / \mathrm{cm} 3$. The phase diagram and densitypressure isotherms for $\mathrm{CO}_{2}$ are, correspondingly, showed in Figures 2.7 and 2.8. Present applications employing scCO2 include coffee decaffeination, drycleaning, chemical extraction and separation, flouro polymerization, low-temperature polymer processing, and biological and pharmaceutical processing.

Among differing types of fillers, which supported natural silicates and clay are excentisively utilized due to its straightforward availability and abundance. The polymer silicates $\mathrm{NCs}$ are in specific category of material of multifunctional and are suffused with the nanoclays with width of a number of nanometers and a whole bunch to thousands of nanometers long. These particles were shaped by polymer intercalation inside the filler platelets. Equally distributed nanoclays of big ratios to provide a large form of extra advantages to the material like optical, mechanical, electrical, thermal and alternate properties of natural.

Nanoclay is derived from MMT which is widely used for the preparation of polymer NC. MMT is a deposit of minerals that has a structure of layer dimensions around $1 \mathrm{~nm}$ thickness and a surface area of specific of 700-800 $\mathrm{m} 2 / \mathrm{g}$ [14-15]

Due to the "cations $(\mathrm{Na}+, \mathrm{K}+, \mathrm{Ca} 2+)$ " within the internal layer, the organic silicates which are layered and hydrophilic and for this reason are in general appropriate with a polymers of hydrophilic, along with poly (vinyl alcohol) (PVA) ensuing in poor clay dispersion in most hydrophobic polymers. The cations inside the galleries between the clay platelets, however, can be changed with organic cations consisting of alkyl ammonium ions to attain silicates of organophilic layers that were mostly have hydrophobic compatibility. The organic cations minimize the energy of surface within the galleries and could boom the distances of interlayer, therefore, changed MMT can extra without difficulty engage along the matrix of polymer, forming exfoliation morphology and improves the association strength in middle of the polymer and clay.There are persevering with efforts exploitation of latest trends in nanotechnology.

The authors "Persico et al." analyzed the feasible utilization of soften combined nylon 6 nanocomposites through exclusive ratio of jajoba/clay oil for cosmetic fabric applications. They analyzed the oil resorption from nanoclay fiber composites with various morphologies. Analysis of the properties and structure of a MMT hybrid/ polypropylene composites and melted fibers of spun explored that better interjection of clay in a matrix of PP advances the spin ability [16]. It 
was asserted that correct intercalation of clay in PP matrix barely decreased the viscous elasticity because of division of linking complication with big phase ratios of platelets of clay, removed the fracture of surface soften also [17], and postponed the complex rate of shear for onset of soften fractures. At the same ratio of draw, fibers with clay had better crystallinities, decrease orientations, and advanced absorption of moisture and dye affinities [16]

PET/clay NC more suitable mechanical and barrier properties [17-19] have been defined previously in the literature. Several workers have attempted to synthesis exfoliated silicate in PET as nicely using melt blending and in situ polymerization methods. Some authors have stated $25 \%$ oxygen permeability discounts for PET/MMT (1 wt \%) by way of using in situ polymerization. A $50 \%$ water vapor permeability reduction for PET/MMT nanocomposite produced by means of soften mixing became additionally suggested [20].Due to high temperatures wanted for processing PET, the PET NC have grow to be principal technological task because of capacity degradation of clay's organic change all through processing [21].There were number of techniques for the polymer NC synthesis which include answer melt intercalation, intercalation in emulsion polymerization, situ polymerization, high-shear mixing and roll milling[22]. For synthesizing a polymer NC, unique crucial factor is the nanoparticles inclusion into the matrix of polymer.

Because of The massive area of surface for each particle volume and the intensely minimum size, leads critical to survive the internal thermodynamics control of aggregation of nanoparticles.

Indeed properly-distributed nanoparticles absolutely gathered to shape patterns whose measure elaborates to scales of length outstrip $1 \mu \mathrm{m}$ [23].

As an end outcome, the number one mission for polymer NC synthesizing is studying the way to distribute proper nanoparticles within a matrix of polymer and eliminate the nanoparticles aggregation.

Outstanding distributions and load transfer of interfacial were normally needed to acquire most fulfilling attributes of the polymer NC which was synthesized. A not unusual approach to boom the chances of dispersion of isotropic was to change the nanoscale filler surface to grow interactions favoring the matrix of polymer and nanofiller while reducing the inter filler enchantment [24].

The method is to combine little substance of polymer or molecules to the nanoclay surface. Furthermore, carbon dioxide of supercritical can efficiently bloat and plasticize the nebulous place of the polymer and be launched from the polymer definitely by depressurizations. Little perticles dissolved in scCO2 may be introduced and accumulated into a matrix of polymer uncovered to one of these answer. $\mathrm{ScCO} 2$ addon the distribution of clay and polymer intercalation inside nanoparticles of clay, indeed if favoring intercalation in middle of the clay and the polymer aren't gift, as within the instance of solely polymers of hydrophobic.[25,26]

Norazlina Hashim et al experimental and theoretical studies of solubility and viscosity of several polymer melts in clay are discussed in detail. The assistance of $\mathrm{scCO} 2$ in clay dispersion and as a foaming agent has been reviewed extensively.[2]

Yang Zhao, Han-Xiong Huang studied The effects of dissolved $\mathrm{ScCO} 2$ on the morphology of nano-CaCO3 and nano-clay were investigated in the polymer nanocomposites prepared using a twin-screw extruder. X-ray diffraction (XRD) and transmission electron microscopy (TEM) were used to characterize the morphology of extruded nanocomposites. The dynamic rheological properties were measured using a dynamic rheometer in the oscillatory mode. Compared to the nanocomposites prepared without the aid of $\mathrm{ScCO} 2$, the nanocomposites with $\mathrm{ScCO} 2$ addition appear to have higher degree of nano-filler dispersion or intercalation/exfoliation. [3]

Quang T. Nguyen, Donald G. Baird is developed a process which uses supercritical carbon dioxide (sc$\mathrm{CO} 2$ ) as a processing aid, to help exfoliate and disperse nanoclay into the polymer matrices. The process relies on rapid expansion of the clay followed by direct injection into the extruder where the mixture is dispersed into the polymer melt. Results from the mechanical properties, rheological studies, and X-ray diffraction (XRD) show that this method represents a significant improvement relative to direct melt blending in single or twin-screw extruders or other methods using sc-CO2. The greatest mechanical property response was a result of directly injecting pre-mixed sc-CO2 and nanoclay into the polypropylene melt during extrusion. It was observed that for concentrations as high as 6.6 wt $\%$ (limited only by present process capabilities),XRD peaks were eliminated, suggesting a high degree of exfoliation. Mechanical properties such as modulus increased by as much as $54 \%$. [4]

S. G. Kazarian review describes research in the applications of supercritical fluids to polymer processing. The ability of supercritical carbon dioxide to swell and plasticize polymers is crucial to the impregnation, extraction, and modification of polymeric materials. This plasticization also reduces viscosity and facilitates the processing of polymers due to lower shear stresses. Spectroscopy plays an important role in 
probing these interactions at a molecular level and to follow in situ the processes of CO2-induced plasticization and the crystallization of polymers. Opportunities exist for improving the processing of many polymeric-based materials ranging from textile to food and biomaterials. The implications of interactions between supercritical carbon dioxide and polymers for drying, dyeing, foaming and extrusion are also discussed with an outlook for further opportunities in this and related areas of polymer processing. [5]

Steven Horsch et al explore a novel supercritical carbon dioxide ( $\mathrm{scCO} 2$ ) processing method that utilizes scCO2 to disperse nano-clays. The structure and properties of the clays and the resultant nanocomposites are characterized using a combination of wide-angle X-ray diffraction (WAXD), scanning electron microscopy (SEM), transmission electron microscopy (TEM), and rheology. Significant dispersion was achieved with dry Cloisite 93A clay, whereas relatively poor dispersion was achieved with dry Cloisite Nap (natural clay). The extent of clay dispersion appears to be dependent on the 'CO2-philicity', which in turn appears to depend on the surface modifications and inter-gallery spacing. The presence of an acidic hydrogen on the surfactant in Cloisite 93A appears to play a strong role in its ' $\mathrm{CO} 2-$ philicity'. The ability to delaminate dry clays is significant because it will likely increase the ability to produce dispersed clay/polymer nanocomposites via melt processing. In addition to delaminating dry clays, we demonstrate that $\mathrm{CO} 2$-phobic Cloisite Nap (natural clay) can be partially dispersed with scCO2, using a $\mathrm{CO} 2$-philic polymer, polydimethylsiloxane (PDMS). The dispersed clay/PDMS nanocomposite shows an order of magnitude increase in the dynamic storage modulus at low frequencies, accompanied by the emergence of a 'solid-like' plateau, characteristic of dispersed nanocomposites with enhanced clay/polymer interactions. [6]

Fengyuan Yang et al, studied Supercritical carbon dioxide (scCO2) dispersion of poly(ethylene terephthalate)/clay nanocomposites: Structural, mechanical, thermal, and barrier properties. Dispersed poly(ethylene terephthalate) (PET)/clay nanocomposites can lead to materials with superior barrier and mechanical properties. PET/clay nanocomposites were prepared by melting extrusion of PET with as-received or supercritical carbon dioxide ( $\mathrm{scCO} 2)$ predispersed CloisiteVR 30B (30B). The predispersion of 30B was assessed by WAXD, SEM, and TGA, and results indicated that $\mathrm{scCO} 2$ processing could predisperse $30 \mathrm{~B}$ and the surface modification of the clay was preserved after processing. The structure of PET/30B nanocomposites was investigated by WAXD and TEM confirming that PET has penetrated into the clays intergalleries and the predispersed clays lead to improved interfacial interaction and homogenous clay dispersion. Both tensile strength and Young's modulus were improved by $12.1 \%$ and $24.9 \%$ respectively, as incorporating of $3 \mathrm{wt} \%$ of scCO2 processed clay. Differential scanning calorimetry (DSC) results indicated that clay particles served as nucleation agent could increase the crystallinity whereas had no impact on melting process. In addition, with the addition of 1 wt $\%$ of predispersed clay, a significant reduction of oxygen permeation (_33\%) was achieved at $238 \mathrm{C}$ and the maximum reduction (44\%) was achieved by adding 3 wt $\%$ processed clay. Moreover,we confirmed the effect of temperature on the permeation of PET/30B nanocomposites depended both on the Arrhenius behavior of the organic phases and tortuous path effects, where improved clay dispersion resulted in a higher effective activation energy.Moreover, the transparency of PET matrix was preserved for all nanocomposites. [7]

M.R. Thompson et al ., examines several new methods for compounding nanocomposite materials by twin screw extrusion that use supercritical $\mathrm{CO} 2$ as a processing aid to produce more highly exfoliated polyolefin-layered silicate nanocomposites than conventional melt intercalation. These methods varied the manner in which the plasticizing behavior of $\mathrm{CO} 2$ influences the surfactant of an organoclay, the compatibilizer, and the matrix during preparation of a polyolefin nanocomposite. The results have shown that targeting $\mathrm{CO} 2$ to the organoclay-compatibilizer interface can improve the extent of intercalation. However, reduced performance was observed when $\mathrm{CO} 2$ was introduced predominantly to the matrix or neat organoclay. In general, the different techniques of addition for $\mathrm{CO} 2$ did bring about greater structural changes to the organoclay, but the stiffness of the resulting materials was lower than simply following a conventional melt intercalation approach.[8]

\section{CONCLUSION}

Compared to the nanocomposites prepared without the aid of $\mathrm{ScCO} 2$, the nanocomposites with $\mathrm{ScCO} 2$ addition appear to have higher degree of nano-filler dispersion or intercalation/exfoliation. [3] The greatest mechanical property response was a result of directly injecting premixed sc-CO2 and nanoclay into the polypropylene melt during extrusion. It was observed that for concentrations as high as $6.6 \mathrm{wt} \%$ (limited only by present process capabilities),XRD peaks were eliminated, suggesting a high degree of exfoliation. Mechanical properties such as modulus increased by as much as 54\%. [4] S. G. Kazarian review describes research in the applications of supercritical fluids to 
polymer processing. The ability of supercritical carbon dioxide to swell and plasticize polymers is crucial to the impregnation, extraction, and modification of polymeric materials. This plasticization also reduces viscosity and facilitates the processing of polymers due to lower shear stresses. Spectroscopy plays an important role in probing these interactions at a molecular level and to follow in situ the processes of CO2-induced plasticization and the crystallization of polymers. Opportunities exist for improving the processing of many polymeric-based materials ranging from textile to food and biomaterials. The implications of interactions between supercritical carbon dioxide and polymers for drying, dyeing, foaming and extrusion are also discussed with an outlook for further opportunities in this and related areas of polymer processing. [6] Dispersed poly(ethylene terephthalate) (PET)/clay nanocomposites can lead to materials with superior barrier and mechanical properties. Differential scanning calorimetry (DSC) results indicated that clay particles served as nucleation agent could increase the crystallinity whereas had no impact on melting process. In addition, with the addition of $1 \mathrm{wt} \%$ of predispersed clay, a significant reduction of oxygen permeation (_33\%) was achieved at $238 \mathrm{C}$ and the maximum reduction (44\%) was achieved by adding $3 \mathrm{wt} \%$ processed clay. Moreover,we confirmed the effect of temperature on the permeation of PET/30B nanocomposites depended both on the Arrhenius behavior of the organic phases and tortuous path effects, where improved clay dispersion resulted in a higher effective activation energy.Moreover, the transparency of PET matrix was preserved for all nanocomposites. [7]

\section{REFERENCES}

[1] Amra Bratovčić*, Amra Odobašić, S. Ćatić, Indira Šestan ,Application of polymer nanocomposite materials in food packaging Croat. J. Food Sci. Technol. (2015) 7 (2)

[2] Norazlina Hashim*,1 and Kamal Yusoh2 ,Supercritical Carbon Dioxide-Assisted Process In Synthesis of Polymer/Clay Melt Macromolecular Research, Vol. 23, No. 5, pp 403-417 (2015)

[3] Yang Zhao, Han-Xiong Huang,Effect Of Supercritical Co2 On Morphology And Rheology Of Polymer Nanocomposites Prepared By Melt Compounding (2006)

[4] Quang T. Nguyen, Donald G. Baird*,An improved technique for exfoliating and dispersing nanoclay particles into polymer matrices using supercritical carbon dioxide, Chemical science (2007)

[5] Steven Horsch, Gulay Serhatkulu, Esin Gulari, Rangaramanujam M. Kannan*Supercritical CO2 dispersion of nano-clays and clay/polymer nanocomposites Polymer Science (2006)

[6] S. G. Kazarian,Polymer Processing with Supercritical Fluids (2000)

[7] Fengyuan Yang,1,2 Christopher Mubarak,3 Robert Keiegel,3 Rangaramanujam M. Kannan1, Supercritical carbon dioxide $(\mathrm{scCO} 2)$ dispersion of poly(ethylene terephthalate)/clay nanocomposites: Structural, mechanical, thermal, and barrier properties Journal of Applied Polymer Science. May 2017

[8] M.R. Thompson,1 Z. Zhuang,1 J. Liu,1 W.R. Rodgers2, Supercritical CO2 as an Exfoliating Aid for Nanocomposite Preparation: Comparison of Different Processing Methodologies POLYM. ENG.pp 2049-2062, 2012

[9] J. Jordan, K. I. Jacob, R. Tannenbaum, M. A. Sharaf, and I. Jasiuk, "Experimental trends in polymer nanocomposites-a review," Materials Science and Engineering:A, vol. 393, pp. 1-11, 2005.

[10] Litchfield DW and Baird DG. The rheology of high aspect ratio nano-particle filled liquids. Rheology Reviews, 2006: $1-60$.

[11] Lucilene Betega de Paivaa LB, Moralesa AR and Diaz FRV. Organoclays: properties, preparation and applications. Applied Clay Science, 2008; 42: 8 $-24$.

[12] Pavlidou S and Papaspyrides CD. A review on polymer-layered silicate nanocomposites. Progress in Polymer Science, 2008; 33: 1119 - 1198.

[13] Şen F, Palancıoğlu $H$ and Aldaş K. Polymeric nanocomposites and their usage areas. Teknolojik Araştırmalar, 2010; 7: 111 - 118.

[14] Kamena, K. (2005). Polyolefins, Houston.

[15] Opalko, R. J. (2008). Evaluation of effects of nanofil nanoclays in the blending of polypropylene and polystyrene. Thesis. Akron, $\mathrm{OH}$.

[16]34Zhang, X., and Yang, M. (2004). Polypropylene/montmorillonite composites and their application in hybrid fiber preparation by melt-spinning. Journal of AppliedPolymer Science, 92, 552-558.

[17] Imai, Y., and Nishinura, S. (2002). High modulus poly(ethylene terephthalate)/Expandable Fluorine Mica Nanocomposites with a novel reactivecompatibilizer. Chemistry of Materials , 14, 477-479.

[18]Zhang, G., and Shichi, T. (2003). PET-clay hybrids with improved tensile strength.Materials Letters, 57, 1858-1862

[19] Tsai, T. Y., and Li, C. H. (2005). Preparation of exfoliated polyester/claynanocomposites. Journal of Advanced Materials, 17, 1769-1773. 
[20] Sanchez-Garcia, M., and Gimenez, E. (2007). Novel PET nanocomposites of interest in food packaging applications and comparative barrier performance with biopolymer nanocomposites. Journal of Plastic Film \& Sheeting, 23, 133-148.

[21] Kim, Sung-Gi. (2007). PET nanocomposite development with nanoscale materials.University of Toledo.

[22] Koo, J. H., Polymer Nanocomposites: Processing, Characterization, and Applications. McGraw-Hill: New York, 2006; p 272.

[23] Schaefer, D. W.; Justice, R. S., How Nano Are Nanocomposites?Macromolecules 2007, 40 (24), 8501-8517.

[24] Crosby, A. J.; Lee, J.-Y., Polymer Nanocomposites: The "Nano" Effect on Mechanical Properties. Polymer Reviews 2007, 47 (2), 217-229.

[25] Herrera-Alonso, M.; Garcia-Leiner, M.; McCarthy, T. J.; Lesser, A. J.,Semicrystalline Polymer Nanocomposites Using Chemically Designed Compatibilizersand Supercritical CO2-Assisted Polymer Processing. PMSE Preprints 2004, 91, 921-922.

[26] Garcia-Leiner, M.; Lesser, A. J., Polymer Nanocomposites Prepared bySupercritical Carbon Dioxide-Assisted Polymer Processing. Polymer Preprints 2004, 45(1), 520-521. 